\title{
Approximated Stiffness Coefficients in Rotor Systems Supported by Bearings with Clearance
}

\author{
Magnus Karlberg \\ Division of Computer Aided Design, Department of Applied Physics and Mechanical Engineering, \\ Luleå University of Technology, 97187 Luleå, Sweden \\ Correspondence should be addressed to Magnus Karlberg, magnus.karlberg@ltu.se
}

Received 29 June 2009; Accepted 9 January 2010

Academic Editor: Kazuhiko Kawaike

Copyright ( 92010 Magnus Karlberg. This is an open access article distributed under the Creative Commons Attribution License, which permits unrestricted use, distribution, and reproduction in any medium, provided the original work is properly cited.

Many kinds of rotating machinery are supported by bearings with clearance, which are further clamped in a supporting structure. When designing such machinery it is important to be able to predict dynamics and hence valid models are needed. Due to gravity, the shaft often vibrates close to a static equilibrium position leading to a possibility to linearise the equation of motion. Although several studies on bearings with clearance exist, there are still no reports on how such clearances affects the stiffness coefficients close to a static equilibrium position. Therefore, analytical expressions for such approximated pedestal stiffness coefficients have been derived in this paper. By using such approximated pedestals in simple rotordynamical models, it was found that the eigenfrequencies decrease significantly with clearance. It is further shown that the approximated pedestal stiffness coefficients only will be valid for vibrations close to the static equilibrium position.

\section{Introduction}

Many kinds of rotating machinery are supported by bearings with clearance, which are further clamped in a supporting structure, that is, pedestals. When designing such machinery mathematical models are needed in order to predict dynamics and thereby avoid unwanted vibrations.

Tiwari et al. [1] showed that both vertical and horizontal ball bearings stiffness decreases with clearance. They further showed that complicated nonlinear motion can occur in systems supporter on ball bearings if the clearance becomes large enough. For a similar rotor system, supported by ball bearings, Mevel and Guyader [2] found different routs to chaos experimentally.

Several studies, both analytical and experimental, on dynamics due to clearance have been carried out by use of a Jeffcott rotor which impacts a rigid stator (snubber ring) supported by springs [3-6]. In these studies, the rotor goes in and out of contact and the piecewise change in stiffness gives a nonlinear system. Chang-Jian and Chen [7] showed that complicated motion can occur in rub-impact
Jeffcott rotor systems supported by oil-film short bearings. Attempts have further been made to find approximate solutions for piecewise linear systems. Karpenko et al. [8] discussed two different approximate methods (one point method and multiple point method) while Kim et al. [9] tested replacing the signum and absolute functions with smoothening functions.

Due to, for example, gravity, many rotating systems will displace in one direction and thereby avoid to loose contact during vibrations, that is, if the unbalance and spin speed is not too high. Childs [10] and Ehrich [11] (1988) found subharmonic responses of similar systems considering gravity and nonsymmetric clearance effects. Further, Ganesan [12] studied stability of the same model as was used by Childs [10].

So far, little has been reported on how the bearing and supporting structure common stiffness depends on clearance in systems subjected by a static load (as gravity) causing the system to vibrate close to a static equilibrium position (s.e.p.). Hence, the objective for this paper is to derive analytical expressions for the approximated pedestal stiffness 


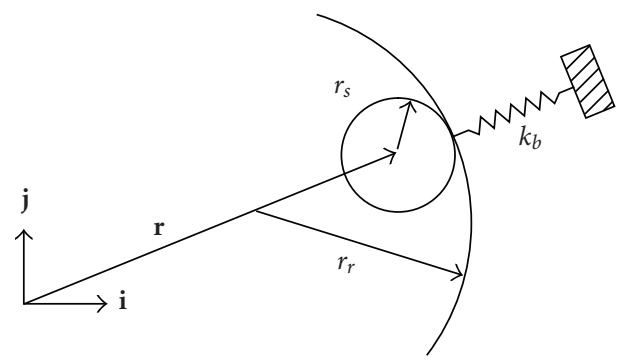

FIGURE 1: Single pedestal unit model.

coefficients in systems with bearing clearance in contact vibrating close to an s.e.p. and to show limitations of such an approximation.

\section{Analysis of Pedestal Stiffness}

To analyse how the pedestal stiffness in rotating machinery depends on clearance in bearings, for example, roller bearings, ball bearings, and so forth. a single pedestal unit is studied see Figure 1.

A shaft is assumed to be in contact with a bearing with clearance which is modeled as a rigid ring which is further supported by a spring, with stiffness coefficient $k_{b}$ which together is referred to as pedestal. The position of the shaft center is given by the vector $\mathbf{r}=x \mathbf{i}+y \mathbf{j}$, and the clearance between the shaft and the stator $\delta=r_{r}-r_{s}$ where $r_{s}$ is the shaft radius and $r_{r}$ the bearing ring radius. When $|\mathbf{r}| \geq \delta$ (i.e., in contact) and the friction in the ring is neglected, the contact reaction force is

$$
\mathbf{F}_{c}=\frac{k_{b}(|\mathbf{r}|-\delta)}{|\mathbf{r}|}(x \mathbf{i}+y \mathbf{j}) .
$$

In many situations a static load is present, for example, due to the weight of the rotor, resulting in shaft whirling around an s.e.p. which here is denoted $\left(x_{\mathrm{se}}, y_{\mathrm{se}}\right)$. The partial derivatives of the contact force given in (1) with respect to $x$ and $y$ are

$$
\begin{aligned}
\frac{\partial \mathbf{F}_{c}}{\partial x}= & k_{b}\left(1-\delta \frac{y_{\mathrm{se}}^{2}}{\left(x_{\mathrm{se}}^{2}+y_{\mathrm{se}}^{2}\right)^{1.5}} \mathbf{i}\right) \\
& +k_{b}\left(1-\delta \frac{x_{\mathrm{se}} y_{\mathrm{se}}}{\left(x_{\mathrm{se}}^{2}+y_{\mathrm{se}}^{2}\right)^{1.5}} \mathbf{j}\right), \\
\frac{\partial \mathbf{F}_{c}}{\partial y}= & k_{b}\left(1-\delta \frac{x_{\mathrm{se}} y_{\mathrm{se}}}{\left(x_{\mathrm{se}}^{2}+y_{\mathrm{se}}^{2}\right)^{1.5}} \mathbf{i}\right) \\
& +k_{b}\left(1-\delta \frac{x_{\mathrm{se}}^{2}}{\left(x_{\mathrm{se}}^{2}+y_{\mathrm{se}}^{2}\right)^{1.5}} \mathbf{j}\right) .
\end{aligned}
$$

From (2), components in different directions can be extracted giving the approximations for the pedestal stiffness coefficients at the s.e.p.

$$
\begin{aligned}
k_{x x} & =\frac{\partial F_{x c}}{\partial x}=k_{b}\left(1-\delta \frac{y_{\mathrm{se}}^{2}}{\left(x_{\mathrm{se}}^{2}+y_{\mathrm{se}}^{2}\right)^{1.5}}\right), \\
k_{x y} & =k_{y x}=\frac{\partial F_{x c}}{\partial y}=\frac{\partial F_{y c}}{\partial x} \\
& =k_{b}\left(\delta \frac{x_{\mathrm{se}} y_{\mathrm{se}}}{\left(x_{\mathrm{se}}^{2}+y_{\mathrm{se}}^{2}\right)^{1.5}}\right), \\
k_{y y} & =\frac{\partial F_{x c}}{\partial x}=k_{b}\left(1-\delta \frac{x_{\mathrm{se}}^{2}}{\left(x_{\mathrm{se}}^{2}+y_{\mathrm{se}}^{2}\right)^{1.5}}\right) .
\end{aligned}
$$

The stiffness matrix for the single pedestal unit shown in Figure 1 is then

$$
K=\left[\begin{array}{ll}
k_{x x} & k_{x y} \\
k_{y x} & k_{y y}
\end{array}\right]
$$

with the displacement vector

$$
\mathbf{x}=\left[\begin{array}{ll}
x & y
\end{array}\right]^{T}
$$

Figure 2(a) shows the stiffness coefficient ratio $k_{x x} / k_{b}$, Figure 2(b) $k_{y y} / k_{b}$ and Figure 2(c) $k_{x y} / k_{b}$ (given by (3)) as a function of $x_{\mathrm{se}}$ for different clearances when $y_{\mathrm{se}}=$ $-1 \cdot 10^{-5} \mathrm{~m}$. The solid line represents $\delta=0 \mathrm{~m}$, dashed line $\delta=2.5 \cdot 10^{-4} \mathrm{~m}$ and dashed dotted line $\delta=5 \cdot 10^{-4} \mathrm{~m}$. Figure 2 shows that the approximated pedestal stiffness coefficients depend on both the amount of clearance and the s.e.p.

\section{Rotordynamics}

To show rotordynamical effects due to bearings with clearance, as shown in Figure 1 and to enable discussions regarding the validity of the approximated pedestal stiffness coefficients one system is modelled in two different ways. An FE-model is used for analyses of the fully nonlinear equation of motion while a Jeffcott rotor is used for analyses of the linearised equation of motion including the approximated pedestal stiffness coefficients close to an s.e.p (see (4)). Notice that all numerical data are given in SI-units.

3.1. The Fully Nonlinear FE-Model. Here, the rotor system is modelled by use of finite elements and lumped matrices (see Figure 3). The shaft consists of two Euler beam elements with the d.o.f. $x, y, \varphi$ and $\theta$ at each node. The $\mathbf{i j}$-plane is perpendicular to the rotational symmetric $z$-axis, $\varphi$ is rotation around the $\mathbf{i}$-axis and $\theta$ rotation around the $\mathbf{j}$-axis. The young's modulus of the Euler beams is set to $E=$ $2 \cdot 10^{15} \mathrm{~N} / \mathrm{m}^{2}$, the density to $\rho=7800 \mathrm{~kg} / \mathrm{m}^{3}$ and the radius of the circular section to $0.1 \mathrm{~m}$, that is, a stiff shaft with low weight. By this assumption, the stiffness is concentrated to the bearings which are of interest in this paper. Node one is 


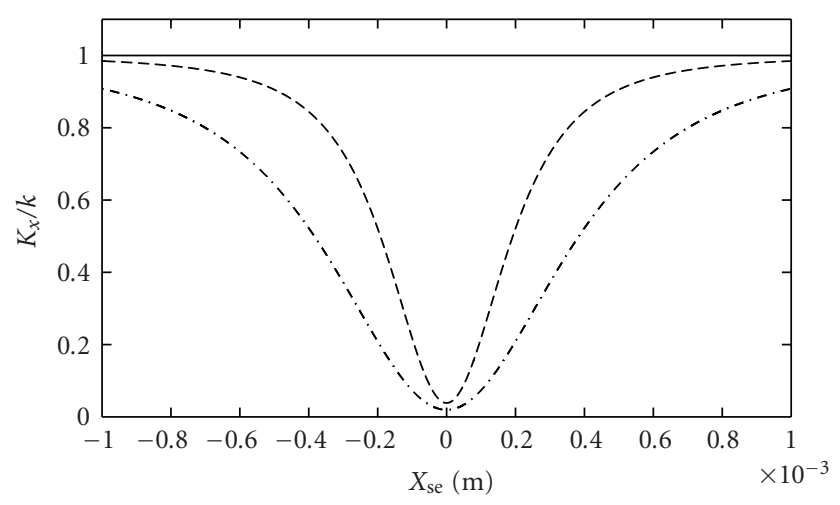

(a)

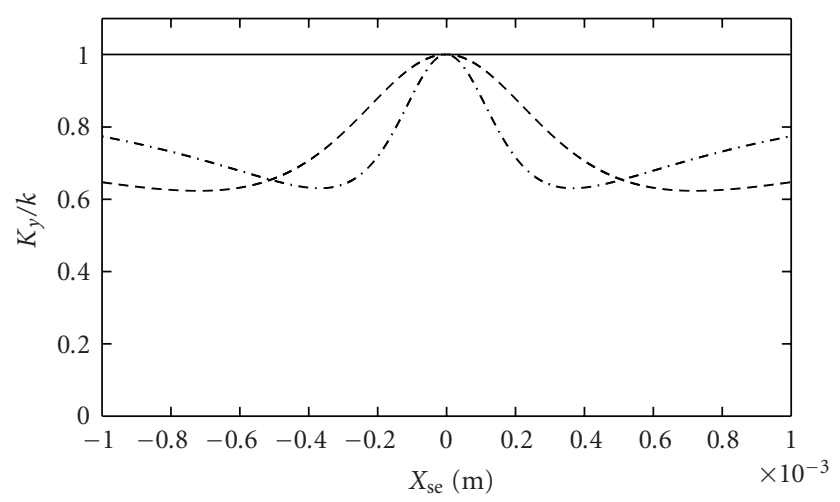

(b)

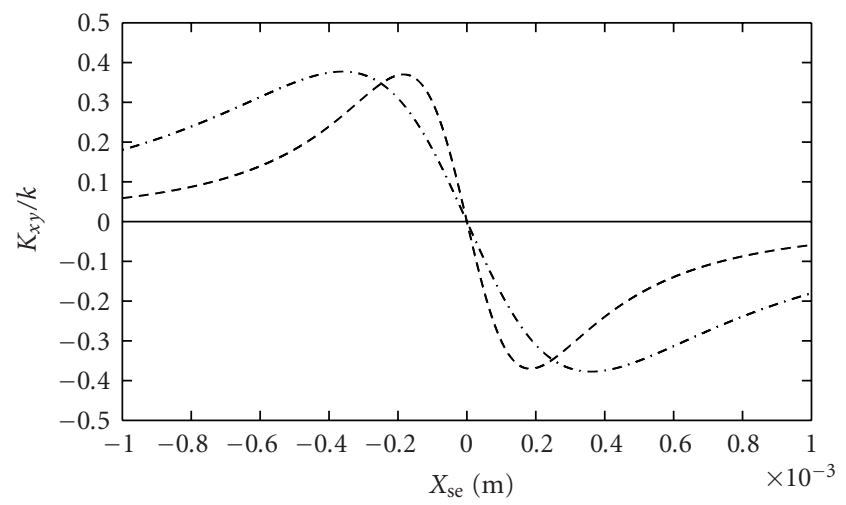

(c)

FIGURE 2: Approximated pedestal stiffness coefficient ratios.

located at the left end, node two 0.5 meter from the left end and node three 1.0 meter from the left end.

The shaft is supported by pedestals with clearance $\delta m$ as shown in Figure 1. The isotropic stiffness coefficient for both pedestals are set to $k_{b}=1 \cdot 10^{9} \mathrm{~N} / \mathrm{m}$. A rigid disc with mass $m=2000 \mathrm{~kg}$, transversal moment of inertia $J_{t}=100 \mathrm{~kg} \cdot \mathrm{m}^{2}$ and polar moment of inertia $J_{p}=200 \mathrm{~kg} \cdot \mathrm{m}^{2}$ is applied at node two. Proportional damping is applied to model, that is, the damping matrix

$$
\mathbf{C}=\alpha \mathbf{M}+\beta \mathbf{K},
$$

where $\alpha=100$ and $\beta=10^{-7}$.

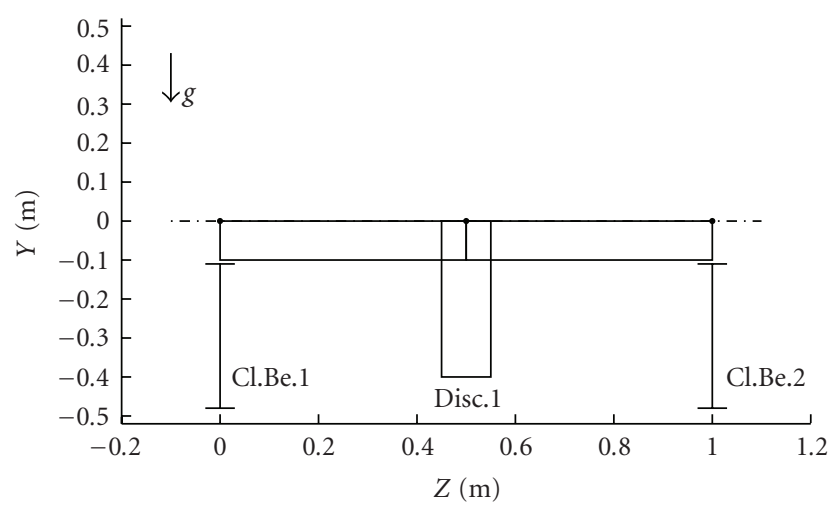

FIGURE 3: FE-model supported by pedestals with clearance.

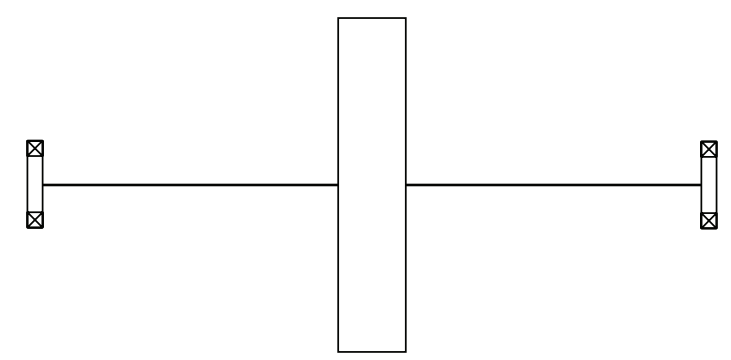

Figure 4: Jeffcott rotor model supported by pedestals with clearance.

3.2. The Jeffcott Rotor Model with Approximated Pedestal Stiffness. Figure 4 shows a Jeffcott rotor with massless shaft and a rigid disc supported by pedestals with clearance $\delta$ as shown in Figure 1. The disc is restricted to translation in $\mathbf{i}$ and $\mathbf{j}$-direction and the massless shaft is considered stiff, that is, the stiffness is only due to the pedestals.

Assuming contact in the bearings $(|r| \geq \delta)$, proportional damping (6) and only unbalance and gravity in $-\mathbf{j}$ direction as external load the governing equation of motion close to the s.e.p. can be approximated by

$$
\begin{gathered}
{\left[\begin{array}{cc}
m_{J} & 0 \\
0 & m_{J}
\end{array}\right]\left[\begin{array}{c}
\ddot{\bar{x}} \\
\ddot{y}
\end{array}\right]+\left(\alpha\left[\begin{array}{cc}
m_{J} & 0 \\
0 & m_{J}
\end{array}\right]+\beta\left[\begin{array}{cc}
2 k_{x x} & 2 k_{x y} \\
2 k_{y x} & 2 k_{y y}
\end{array}\right]\right)\left[\begin{array}{c}
\dot{\bar{x}} \\
\dot{\bar{y}}
\end{array}\right]} \\
+\left[\begin{array}{cc}
2 k_{x x} & 2 k_{x y} \\
2 k_{y x} & 2 k_{y y}
\end{array}\right]\left[\begin{array}{l}
\bar{x} \\
\bar{y}
\end{array}\right]=\left[\begin{array}{l}
m_{J} \gamma \Omega^{2} \sin (\Omega t) \\
m_{J} \gamma \Omega^{2} \cos (\Omega t)
\end{array}\right],
\end{gathered}
$$

where $\Omega$ is the spin speed, $\gamma$ the eccentricity and the coordinates $\bar{x}$ and $\bar{y}$ are displacements in $\mathbf{i}$ - and $\mathbf{j}$-direction with origin in the s.e.p. $\left(x_{\mathrm{se}}, y_{\mathrm{se}}\right)$. The mass of the disc together with the FE-model shaft mass (in order to make the two models comparable) is denoted $m_{J}$.

3.3. Case 1: No Shaft Rotation. For both the Jeffcott and the FE-model, due to gravity $g=-10 \mathbf{j} \mathrm{m} / \mathrm{s}^{2}$, the shaft will displace into an s.e.p.

$$
x_{\mathrm{se}}=0 \mathrm{~m}, \quad y_{\mathrm{se}} \approx-\left(\frac{m_{J} \cdot g}{2 k_{b}}\right)-\delta \mathrm{m}
$$




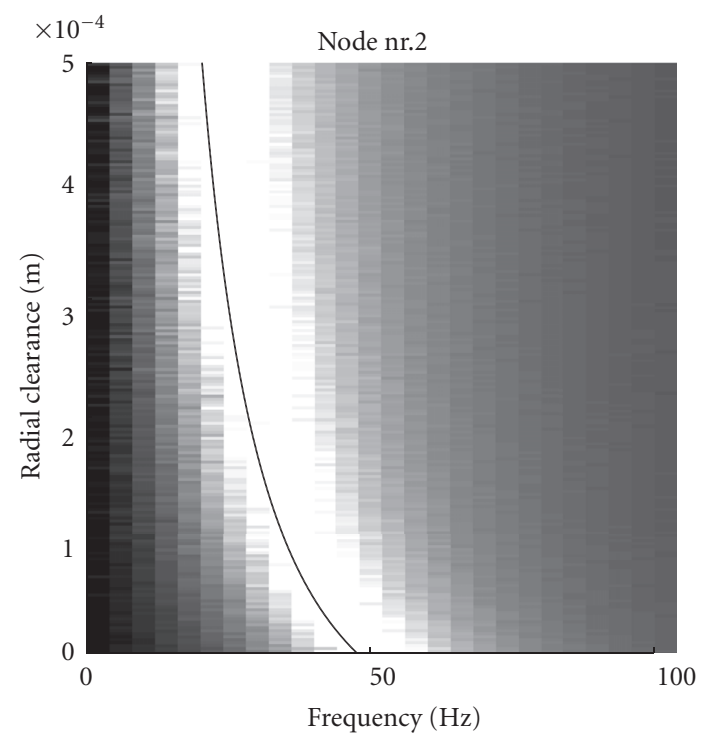

FIGURE 5: Eigenfrequency and transfer function as function of clearance at zero spin speed.

in the bearings. At this s.e.p. Figure 2 shows that the stiffness coefficients $k_{y y}=k_{b}$ and that $k_{x y}=k_{y x}=0$ for every clearance $\delta$ while $k_{x x}$ is linearly dependent of $\delta$.

Since the FE-model has a nonlinear equation of motion, an eigenvalue analysis cannot be performed. However, by applying a random force of $1000 \mathrm{~N}$ in $\mathbf{i}$-direction at the disc node, a transfer function can be derived.

Figure 5 shows the transfer function of the FE-model (for velocity) and the corresponding eigenfrequency of the Jeffcott model for vibrations in $\mathbf{i}$-direction as function of the clearance close to the s.e.p. given by (8) when the spin speed $\Omega=0 \mathrm{~Hz}$. The black dashed line is the eigenfrequencies derived from (7), that is, the Jeffcott rotor, while the black and white area is the transfer function of the FEmodel. White indicates large vibration amplitudes and black indicates low vibration amplitudes. Figure 5 shows that at $\Omega=0 \mathrm{~Hz}$ the eigenfrequencies in $\mathbf{i}$-direction of the Jeffcott model decreases from $50 \mathrm{~Hz}$ to $25 \mathrm{~Hz}$ as the clearance is increased from 0 to $0.5 \mathrm{~mm}$. The eigenfrequencies of the Jeffcott model further coincides with the large amplitudes (resonances) in the transfer function derived from the FEmodel.

3.4. Case 2: Shaft Rotation at Small Amplitudes. In this case, the s.e.p. are given in (8) (i.e., due to gravity $g=-10 \mathbf{j ~ m} / \mathrm{s}^{2}$ ). The shaft rotates with a spin speed $\Omega=30 \mathrm{~Hz}$ and the unbalance is furthermore set to $\gamma=1 \cdot 10^{-7} \mathrm{~m}$.

Figure 6 shows the unbalance response displacement orbit in the ij-plane for Case 2. The solid line represents the FE-model while the dashed line represents the Jeffcott model approximation. Both models give similar elliptical shape of the orbit although the Jeffcott model over predicts the vibration amplitude with a few percent.

Figure 7 shows the maximum amplitude in $\mathbf{i}$-direction at steady state as a function of the spin speed. The solid line

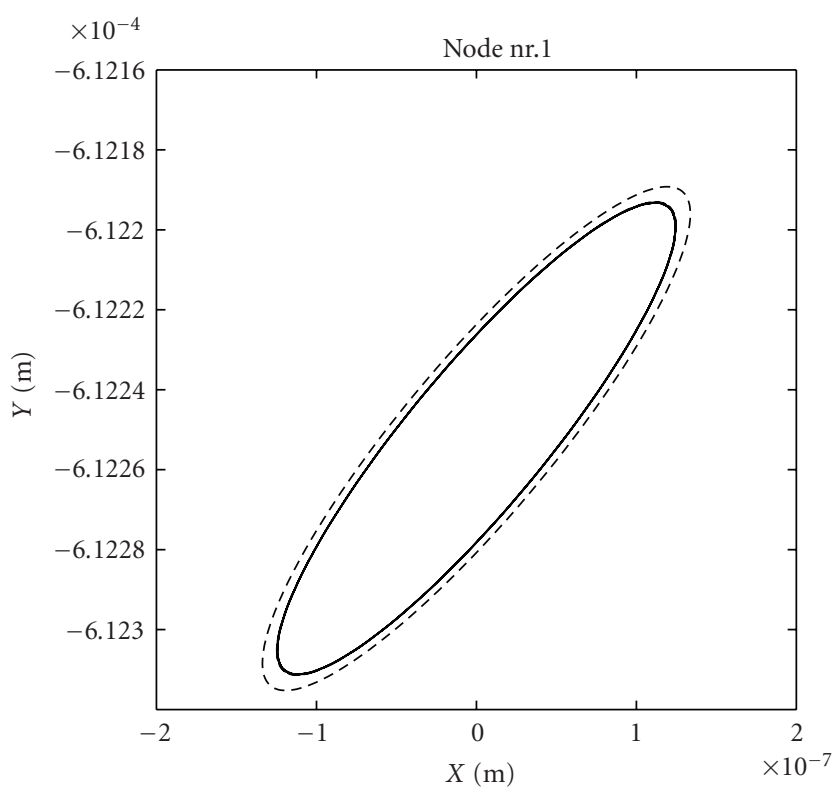

Figure 6: Displacement orbits in Case 2.

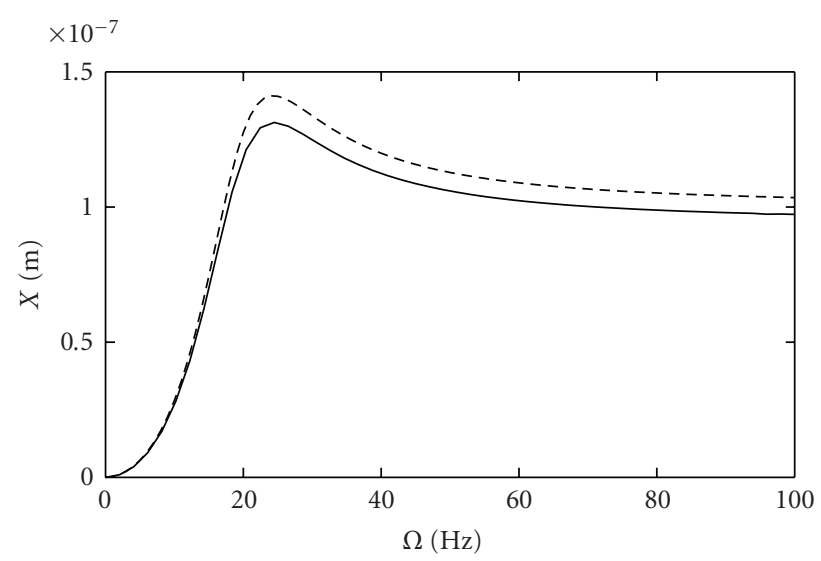

Figure 7: Maximum amplitude as function of spin speed.

represents the FE-model while the dashed line represents the Jeffcott model. This figure shows that the error of the Jeffcott model increases with vibration amplitude, that is, with increased distance from the s.e.p.

3.5. Case 3: Shaft Rotation at Large Amplitudes. In this case, the same set of parameters as for Case 2 has been used except for the unbalance which in this case has been increased to $\gamma=2 \cdot 10^{-4} \mathrm{~m}$.

Figure 8 shows the unbalance response displacement orbit in the ij-plane for Case 3. The solid line represents the FE-model, the dashed line represents the Jeffcott model and the dashed dotted line the clearance (to show that the orbit is in contact). The linearised Jeffcott model still gives an elliptic orbit while the FE-model gives a somewhat distorted elliptical orbit due to the nonlinearity of the clearance. 


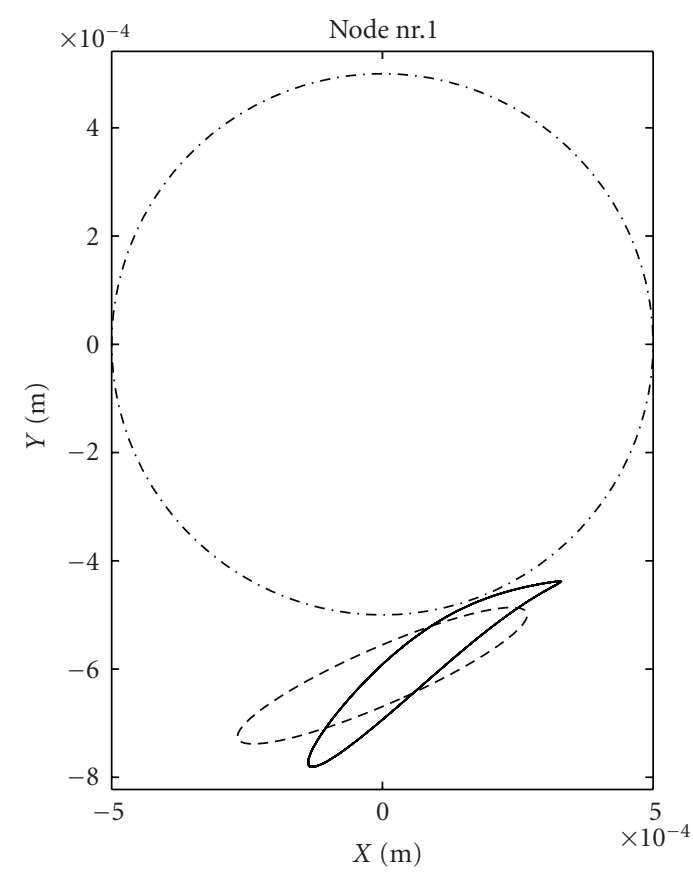

FIGURE 8: Displacement orbits in Case 3.

\section{Discussion and conclusions}

The approximated stiffness coefficients close to an s.e.p. for bearings with clearance derived in this paper requires contact between the shaft and the bearing and that the friction in the contact can be neglected. It is further assumed that the shape of the contact structure (see Figure 1) is remained during contact. These assumptions require that the supporting structure, in which the bearing have been clamped, is significantly weaker than the contact stiffness itself, which is the case, for example, in several rotating machinery supported by roller bearings or ball bearings.

The analyses of a single pedestal unit show that in contact, for example, due to gravity, approximated stiffness coefficients can be derived which depends on both the s.e.p. and the clearance (see (3)). Figure 2 shows how the stiffness coefficient ratio, that is, stiffness coefficients divided by the pedestal stiffness, depends on $x_{\text {se }}$ for different clearances when $y_{\text {se }}=-1 \cdot 10^{-5} \mathrm{~m}$. At no clearance, as expected, the stiffness ratio $k_{x x} / k_{b}=1$ and $k_{y y} / k_{b}=1$ while the cross coupling term $k_{x y} / k_{b}=0$. However, $k_{x x} / k_{b}$ and $k_{y y} / k_{b}$ decreases with the clearance while the absolute value of the cross coupling stiffness increases (it changes sign depending on which side of on the $\mathbf{j}$-axis that is considered).

It can be stated that the clearance give raise to a significant decrease of eigenfrequencies. At no rotation ( $\Omega=0 \mathrm{~Hz}$ ), the eigenfrequencies of the Jeffcott model (and the frequencies of the large amplitudes of the FE-model) decreased with $50 \%$ when the clearance was increased from 0 to $0.5 \mathrm{~mm}$ (see Figure 5). It can also be stated that the approximated stiffness coefficients can be used to predict the dynamics close to the s.e.p. (see Figures 5, 6, and 7). However, for larger vibration amplitudes, the Jeffcott model deviates from the fully nonlinear FE-model (see Figure 8). This means that the validity of the approximated stiffness coefficients decreases with increased vibration amplitudes. Hence, this paper provides analytical expressions for the stiffness coefficients that can be used in rotating machinery models and thereby avoiding fully nonlinear equations. However, these stiffness coefficients will only be valid for vibrations in full contact close to the static equilibrium position.

\section{Acknowledgment}

The work presented in this paper is done within a project in The Faste Laboratory which is financed by Luleå University of Technology together with industrial partners.

\section{References}

[1] M. Tiwari, K. Gupta, and O. Prakash, "Effect of radial internal clearance of a ball bearing on the dynamics of a balanced horizontal rotor," Journal of Sound and Vibration, vol. 238, no. 5, pp. 723-726, 2000.

[2] B. Mevel and J. L. Guyader, "Experiments on routes to chaos in ball bearings," Journal of Sound and Vibration, vol. 318, no. 3, pp. 549-564, 2008.

[3] E. V. Karpenko, E. E. Pavlovskaia, and M. Wiercigroch, "Bifurcation analysis of a preloaded Jeffcott rotor," Chaos, Solitons and Fractals, vol. 15, no. 2, pp. 407-416, 2003.

[4] E. V. Karpenko, M. Wiercigroch, and M. P. Cartmell, "Regular and chaotic dynamics of a discontinuously nonlinear rotor system," Chaos, Solitons and Fractals, vol. 13, no. 6, pp. 12311242, 2002.

[5] S. Popprath and H. Ecker, "Nonlinear dynamics of a rotor contacting an elastically suspended stator," Journal of Sound and Vibration, vol. 308, no. 3-5, pp. 767-784, 2007.

[6] E. V. Karpenko, M. Wiercigroch, E. E. Pavlovskaia, and R. D. Neilson, "Experimental verification of Jeffcott rotor model with preloaded snubber ring," Journal of Sound and Vibration, vol. 298, no. 4-5, pp. 907-917, 2006.

[7] C.-W. Chang-Jian and C.-K. Chen, "Chaos of rub-impact rotor supported by bearings with nonlinear suspension," Tribology International, vol. 42, no. 3, pp. 426-439, 2009.

[8] E. V. Karpenko, M. Wiercigroch, E. E. Pavlovskaia, and M. P. Cartmell, "Piecewise approximate analytical solutions for a Jeffcott rotor with a snubber ring," International Journal of Mechanical Sciences, vol. 44, no. 3, pp. 475-488, 2002.

[9] T. C. Kim, T. E. Rook, and R. Singh, "Effect of smoothening functions on the frequency response of an oscillator with clearance non-linearity," Journal of Sound and Vibration, vol. 263, no. 3, pp. 665-678, 2003.

[10] D. W. Childs, "Fractional-frequency rotor motion due to nonsymmetric clearance effects," Journal of Engineering for Power, vol. 104, no. 3, pp. 533-541, 1982.

[11] F. F. Ehrich, "High order subharmonic response of high speed rotors in bearing clearance," Journal of Vibration, Acoustics, Stress and Reliability in Design, vol. 110, no. 1, pp. 9-16, 1988.

[12] R. Ganesan, "Dynamic response and stability of a rotorsupport system with non-symmetric bearing clearances," Mechanism and Machine Theory, vol. 31, no. 6, pp. 781-798, 1996. 

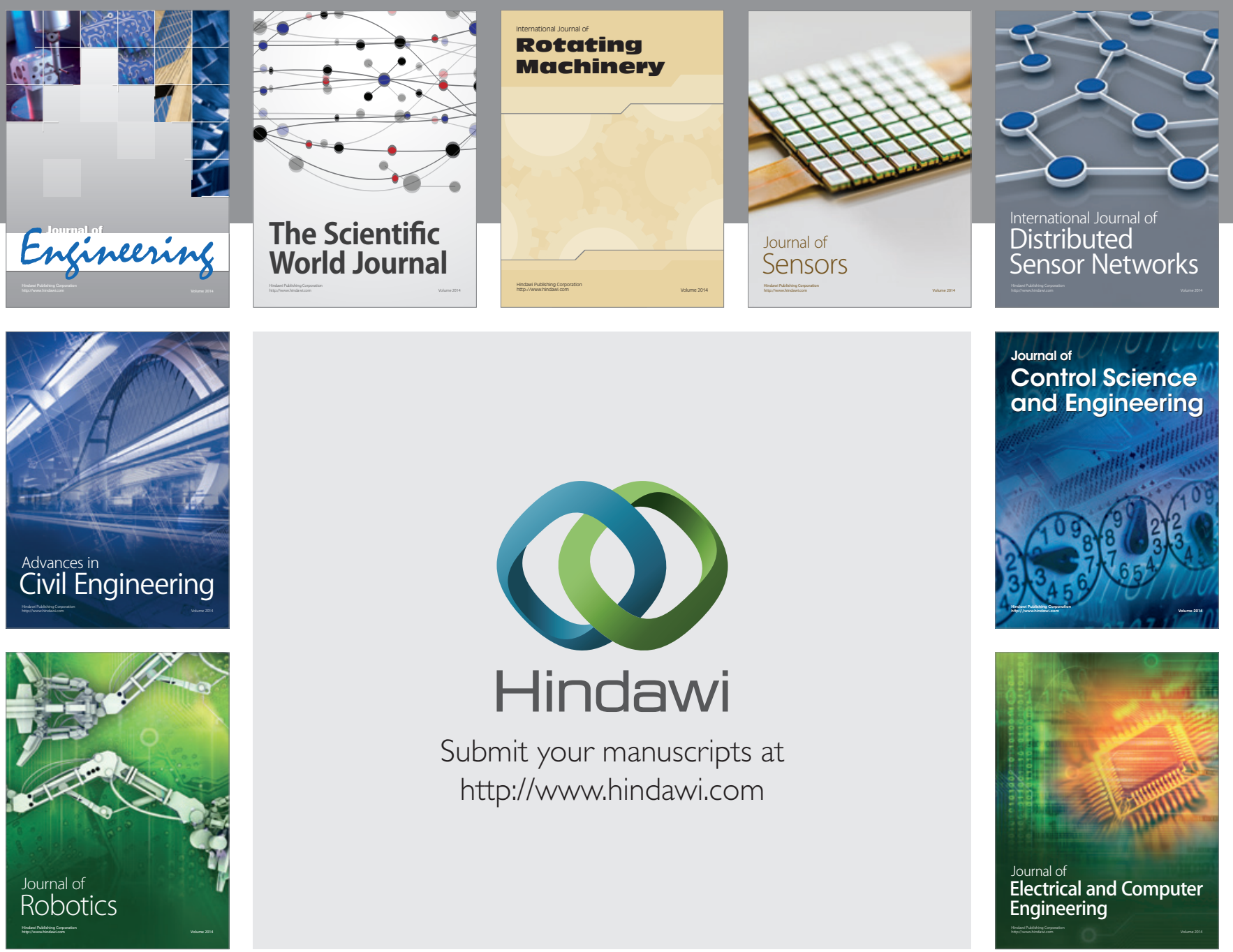

Submit your manuscripts at

http://www.hindawi.com
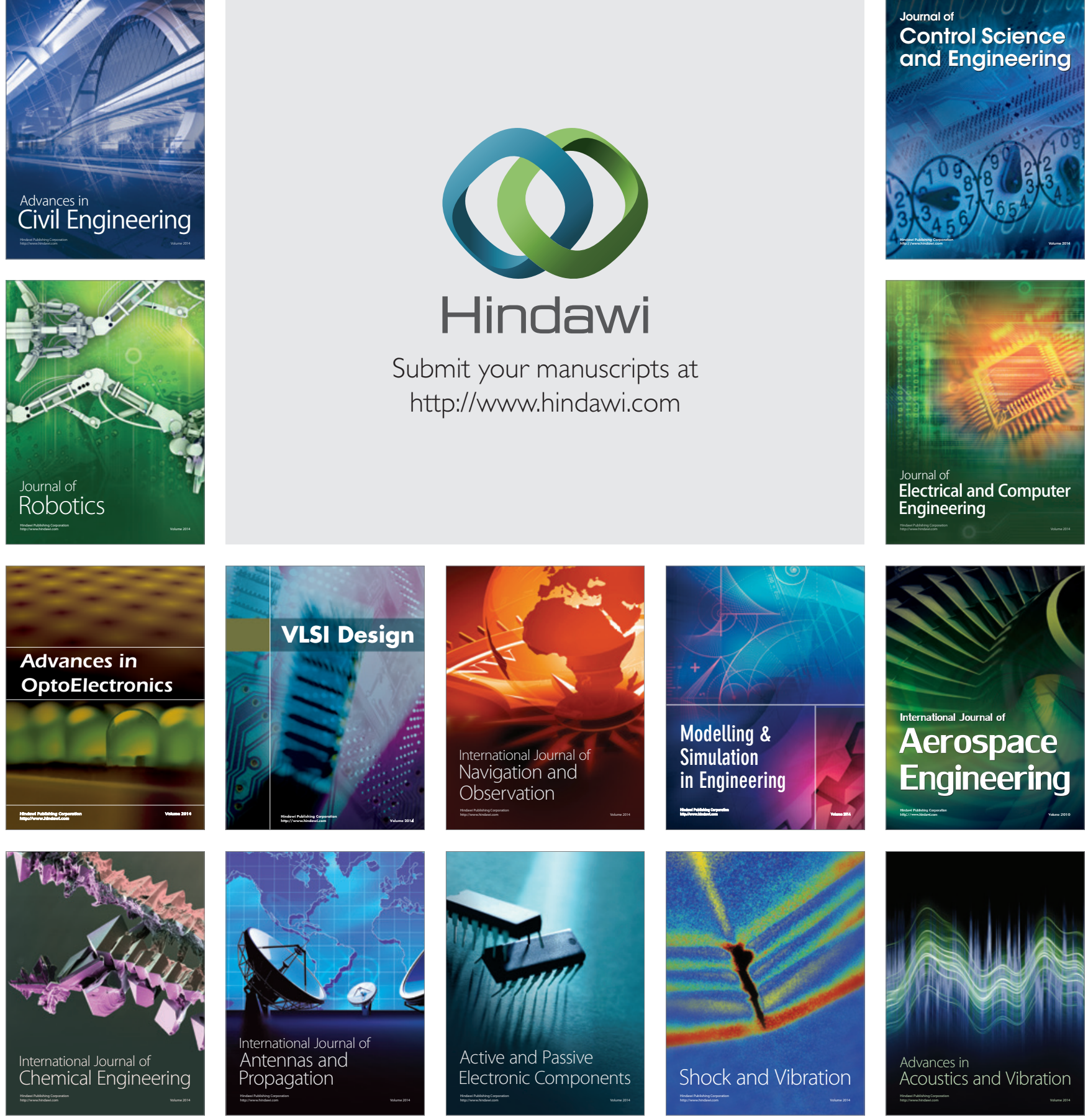